\title{
In search of cosmic order: Astronomy and culture in Ancient Egypt
}

\author{
Juan Antonio Belmonte \\ Instituto de Astrofísica de Canarias, \\ 38205 La Laguna, Tenerife, Spain \\ email: jba@iac.es
}

\begin{abstract}
Over the past five years, the Egyptian-Spanish Mission on the archaeoastronomy of ancient Egypt and its collaborators has been performing an ambitious scientific project with the aim of studying the cosmovision of the ancient civilization of the pharaohs. Part of the project has consisted of a re-analysis of the iconographic and historical sources that has allowed, among other things, a reassessment of the calendar theory and a new proposal for the sky-maps of ancient Egypt. For various reasons, Archaeoastronomy has not been one of the favourite disciplines of egyptologists in the past. Probably because of this, important questions such as the orientation of Egyptian temples and the relevance of astronomy in this respect had never been afforded with the requisite seriousness and depth. Our work has had among its various priorities, the solution of this problem. In order to achieve this, our team has so far measured the orientation of some 330 temples in the Valley, the Delta, the Oases and the Sinai. The aim is to find a correct and almost definitive answer to the question of whether the ancient Egyptian sacred constructions were astronomically aligned or not. Our results may provide an affirmative answer.
\end{abstract}

Keywords. Egypt, archaeoastronomy, alignments, Sirius, Canopus, Ursa Major

\section{Introduction}

Were the temples of the ancient Egyptian civilization astronomically orientated? This is a very important question that has been far from being resolved (see e.g. Badawy 1968). More recently, Wilkison (2000) clearly stated that most commonly temples built along the Nile were oriented on an east-west axis, according to local cardinal directions as determined by the river, so local topography would be the determining reason for temple orientation. However, he also pointed out that on occasions, orientation towards the sun or important stars was definitely the priority, and this principle may be more important than is often recognized. It is the goal of this short essay to show that solar and stellar orientations were much more common in ancient Egypt than had previously been suspected. To achieve this, the author joined efforts in 2004 with his Egyptian colleagues Mosalam Shaltout and Magdi Fekri and together inaugurated a project with the main objective of putting the study of ancient Egyptian archaeoastronomy on the footing it deserves in the context of present-day Egyptology. An Egyptian-Spanish Mission was created under the auspices of the Egyptian Supreme Council of Antiquities with the aim of measuring the orientation of the vast majority of ancient buildings, notably the temples, across Egypt, within a reasonable period of time. The purpose was to obtain sufficient fieldwork data that could prove, or disprove, through statistical studies, all the speculations concerning temple orientation from both the topographical and the astronomical point of view.

Hence, during the last years, our mission and its collaborators has been performing an ambitious scientific project with the aim of studying the cosmovision of the ancient 
civilization of the pharaohs. Part of the project has consisted of a re-analysis of the iconographic and historical sources that has allowed, among other things, a reassessment of the calendar theory, challenging old-fashioned paradigms (see e.g. Belmonte 2009), or a new proposal for the sky-maps of ancient Egypt (see Lull \& Belmonte 2006, 2009). Indeed, the most expensive part of the project, in time, effort and resources, has been the five campaigns devoted so far to measuring the orientation and studying the spatial location of ancient monuments across the Nile Valley and beyond. More than 500 pyramids, hypogea, chapels, sanctuaries or small and large temples have been measured so far. The fieldwork in successive campaigns was organized geographically but also with the intention of testing previous results with new exercises. Accordingly, the first campaign was devoted to Upper Egypt, the second to Middle Egypt, the third to the Oases of the Western Desert, the fourth to Lower Egypt, and the fifth with the fundamental aim of completing the sample and making some further tests. Four successive papers (Shaltout \& Belmonte 2005; Belmonte \& Shaltout 2006; Shaltout, Belmonte \& Fekri 2007; Belmonte, Shaltout \& Fekri 2008, hereafter Papers 1, 2, 3 and 4 respectively) have already been published about the temples in which, stage by stage, we have analysed the relationship of temple orientation and their location within the local landscape, understanding landscape in its broadest meaning of both terrestrial and celestial (astronomical orientations) aspects. Our studies have demonstrated that both components were necessary and indeed intimately correlated. A comprehensive summary has recently been published in Belmonte, Shaltout \& Fekri (2009). In five campaigns, we have so far completed a sample of 330 temples of ancient Egyptian culture in Egypt herself. We have made a revision of most of the relevant archaeological literature and this sample contains data of more than $90 \%$ of all the temples to be found nowadays in Egypt. We consider our sample to be statistically representative beyond any doubt and we are convinced that new data will only serve to reinforce our results (see, for example, Belmonte et al. 2010). Figure 1 presents the orientation histogram of all our data as presented in Papers 1, 2, 3 and 4 . The histogram presents relative frequency versus azimuth and clearly shows two peaks which are statistically significant at $91^{\circ}$ and $117^{\circ}$.

The first is connected with east and we will further discuss it extensively. The second is the azimuth for winter solstice sunrise at the time of the pharaohs for latitudes near ancient Thebes (c. $\left.26^{\circ} \mathrm{N}\right)$. There are two peaks at $134^{\circ}$ and $45^{\circ}$ which surely correspond to NE and SE directions of the compass. Indeed, all these azimuths have an intrinsic astronomical meaning. Hence, the simplest analysis of our data, the rough plotting of azimuth versus relative frequency positively answers the question that was the basis of our project, whether the temples of ancient Egypt were astronomically orientated or not.

\section{Discussing astronomical orientations}

In Paper 3, we proposed for the first time in the study of ancient Egypt that a number of families or patterns of orientations of ancient temples could be identified. These were the result of an analysis performed to the histogram of the absolute value of the declination of only 90 monuments, mostly of Lower Egypt. Indeed, it was worth repeating the exercise with the existing complete sample of 330 temples. The results of the experiment are presented in Fig. 2, Panel (a), and are very suggestive.

As a matter of fact, eleven peaks are significant in the plot and we believe that they correspond to seven possible different families of astronomical alignments of Egyptian temples. These could be classified as follows (same Roman numbers as in Fig. 2):

I The eastern (or equinoctial) family. The peak corresponds to an orientation to the equinoctial sun when the disk has completely risen above the horizon. This might imply 


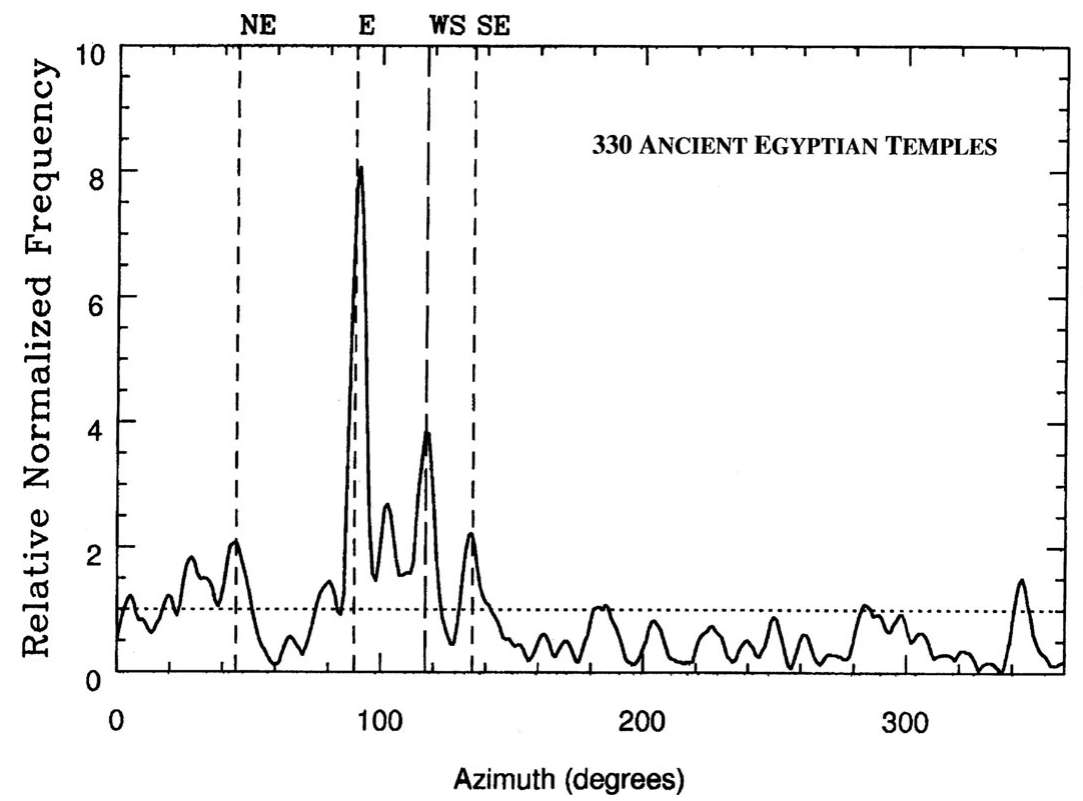

Figure 1. A test of the astronomical hypothesis. Orientation (azimuth) histogram of the 330 temples measured during our first five campaigns in Egypt between February 2004 and December 2006. Although there are temples orientated to each sector of the horizon, there are obviously clear preferences with statistically significant peaks at near due-East (equinoctial sunrise and/or due-north orientations later skewed by $90^{\circ}$ ) and winter solstice (WS) sunrise. There are also clear peaks at NE and SE. These are indisputable evidences of intentional astronomical orientations.

that the ancient Egyptians were able to determine the day of the equinoxes with a reasonable precision. However, another solution is possible. This family could be the result of an orientation in the Meridian line (probably to due-north) and later the gate of the temple would have been open by establishing the perpendicular through standard topographic techniques. The pyramid complexes could be the paradigmatic example of such a procedure (see Fig. 3), where the N-S axis of the pyramid would have been the first element of the construction obtained in the foundation ceremonies (see, e.g., Spence 2000). We can find arguments in favour of one or other alternative and the probable solution is that both kind of monuments ought to be included within the group.

II The solstitial family. This group is dominated by a series of temples orientated to sunrise at the winter solstice (see Fig. 4), although other solstitial orientations have also been documented. Associated to this family, we have the peculiar II $\perp$ peak. This last group of temples includes monuments whose axis was obtained by rotating $90^{\circ}$ anticlockwise a previous orientation determined by winter solstice sunrise.

III The seasonal sun family. We propose the idea that this family has also a solar origin. This group of temples corresponds to monuments orientated to a peculiar interval of declinations related to the seasons of the tropic year and the civil calendar of ancient Egypt, hence the name (see Fig. 5).

IV The Sopdet or Sothic family. The star Sopdet, our Sirius, the brightest star of the ancient Egyptian skies, was very important as the Harbinger of the actual Flooding at least from the Middle Kingdom onwards, when the phenomenon of its heliacal rising (Peret Sopdet) is mentioned in the hieroglyphic texts on several occasions, although its name is also largely mentioned in the Pyramid Texts within the context of the stellar 


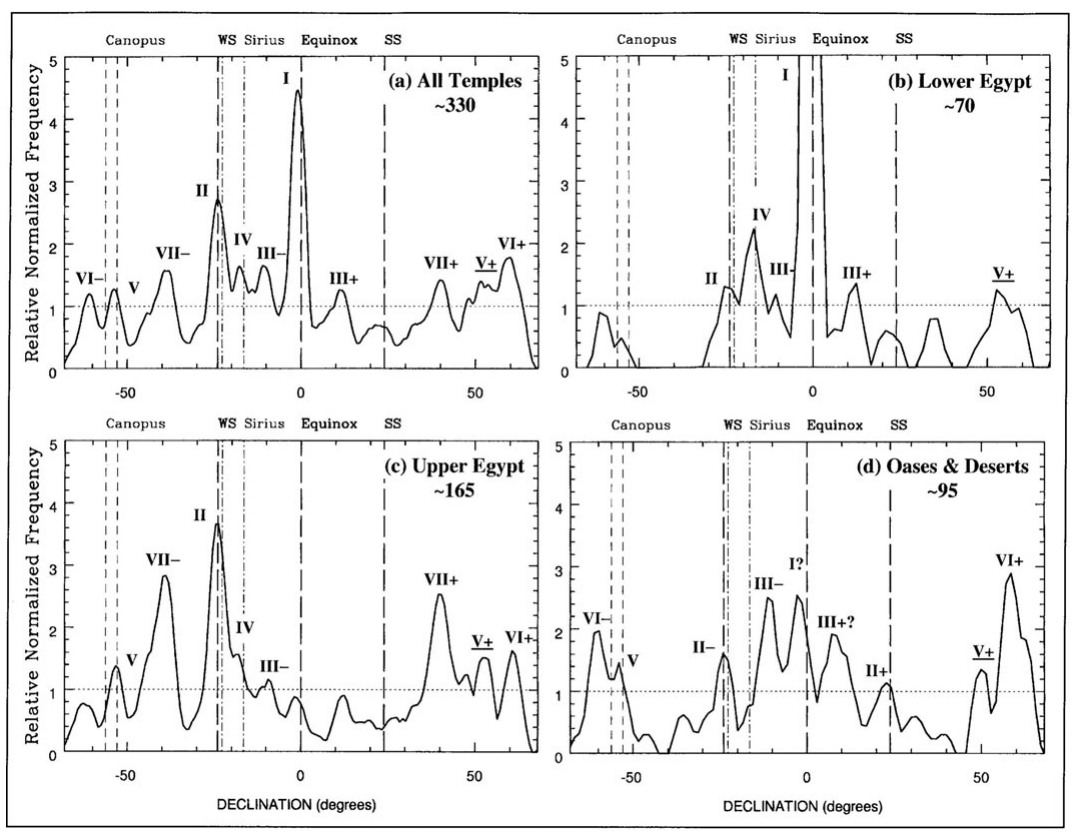

Figure 2. Histogram of declination of the temples of ancient Egypt versus geography. (a) Total histogram of our sample of 330 temples showing the 7 families of orientation, including those like III, VI and VII with positive and negative subfamilies. (b) Temples of Lower Egypt, from Meidum to the sea. (c) Temples of Upper Egypt and Lower Nubia, until Abu Simbel. (d) Temples of the oases and deserts of ancient Egypt. The series of data plotted in panels $(b),(c)$ and $(d)$ are independent of each other. However, the continuity of different traditions, like the solstitial (II), across all Egyptian geography, is obvious. In panel (b), the peak of family I rises to more than 12 but has been cut to keep the same scale in the different plots

eschatology of the Old Kingdom Krauss (1997); Maravelia (2006). This could have been reflected in the architecture and the local landscape (see, for example, Fig. 6).

V The Canopus family. The stellar interpretation of this family is more complicated because we can not prove the importance of Canopus for the ancient Egyptians, notwithstanding the fact that it was their second brightest star in the sky. However, several temples could have been orientated accordingly (see Fig. 7).

VI The Meridian (or northern) family. This clearly speaks of the great importance of near-Meridian, not to say precise N-S, orientations in ancient Egypt. We support the idea that this northern custom was effectively achieved through orientations to certain configurations of stars near the celestial pole, and that the circumpolar constellation of Meskhetyu would be the most appropriate target for this purpose (see Fig. 6). Meskhetyu, the Plough, undoubtedly was one of the most important asterisms of Egyptian religion since at least the Old Kingdom, if not earlier, where it appears in the Pyramid Texts as the imperishable star per excellence, to the Ptolemaic Period when it is profusely mentioned in connection to temple orientation (see e.g. Belmonte, Molinero \& Miranda 2009).

VII The family of inter-cardinal (or quarter cardinal) directions. It is defined by those temples with an orientation close to the SE-NW and SW-NE lines and we believe that it is a subgroup of a cardinal super-family (see Fig. 8). It is our contention that this orientation was achieved by the primary determination of a north alignment, which axis was later rotated by either 45 or 135 degrees clockwise for temples open to the east side 


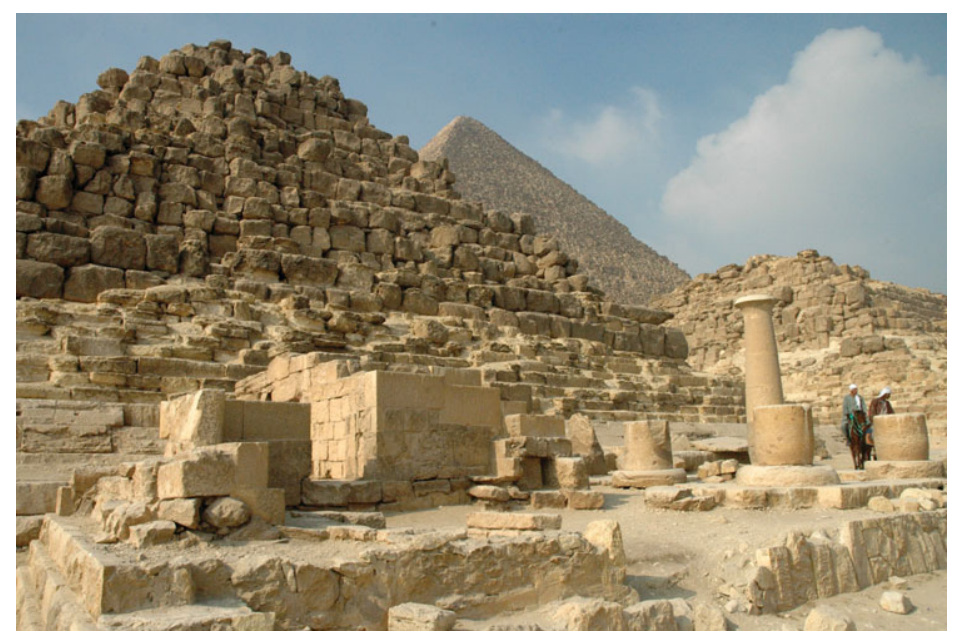

Figure 3. The temple of Isis Lady of the Pyramids constructed in the Late Period over the ruins of the cultic chapel of the pyramid of Henutsen (G1-c). The pyramid of Khufu can be seen in the foreground. Following the pyramid general layout, the temple is equinoctially orientated. Photograph (c) J. A. Belmonte.

of the horizon, which are the most frequent, or anticlockwise for temples open to the west side of the horizon. By performing such an action, simultaneous astronomical and Nile orientations could be achieved in several cases (Belmonte, Shaltout \& Fekri 2009).

In summary, by defining these seven families, we have shown the importance of astronomical orientations in ancient Egypt. However, one advantage of having such a great volume of data at our disposal is that it becomes possible to perform comparative analyses with independent series. We have imagined two such experiments, one with the data of temples separated by historical period and another by geographical location. The results of the first are presented in Fig. 9, where panel (a) shows again for comparison the declination histogram of the whole series, panel $(b)$ for the most ancient temples from the early dynastic period to the Old and Middle Kingdoms, panel (c) is for the temples of the New Kingdom and the Late Period up to the Persian conquest and, finally, panel (d) from that moment to the end of the pharaonic era, including the Ptolemaic and Roman periods.

The results of the study by geographical location are presented in Figure 2. Panel (a) is for all the land, panel (b) stands for the temples of Lower Egypt from the province of the White Wall (area of Memphis) to the sea, panel (c) is for classical Upper Egypt, including what today we know as Middle Egypt and also Lower Nubia up to the Sudanese border, and panel $(d)$ shows the data of all the temples of the deserts and oases of Egypt from Siwa to the Sinai. The three series of both analyses have a large enough number of monuments for the comparison between the different series to be considered realistic and statistically significant. The comparative analysis will be performed by families of orientations and we will try to recognize effects such as possible evolutions, period specialization or geographical peculiarities.

Family $\mathrm{I}$ is very interesting. Indeed it is never appropriately working as equinoctial as may clearly be seen in the successive panels of Figs. 2 and 9 . This justifies the fact that we prefer to simply call it the eastern (cardinal) family. It appears in the oldest periods and predominantly in Lower Egypt. Certainly, it is mostly related to the pyramid complexes of the Old and Middle Kingdom. It shows values of the declination of -0.75 and -0.25 , 

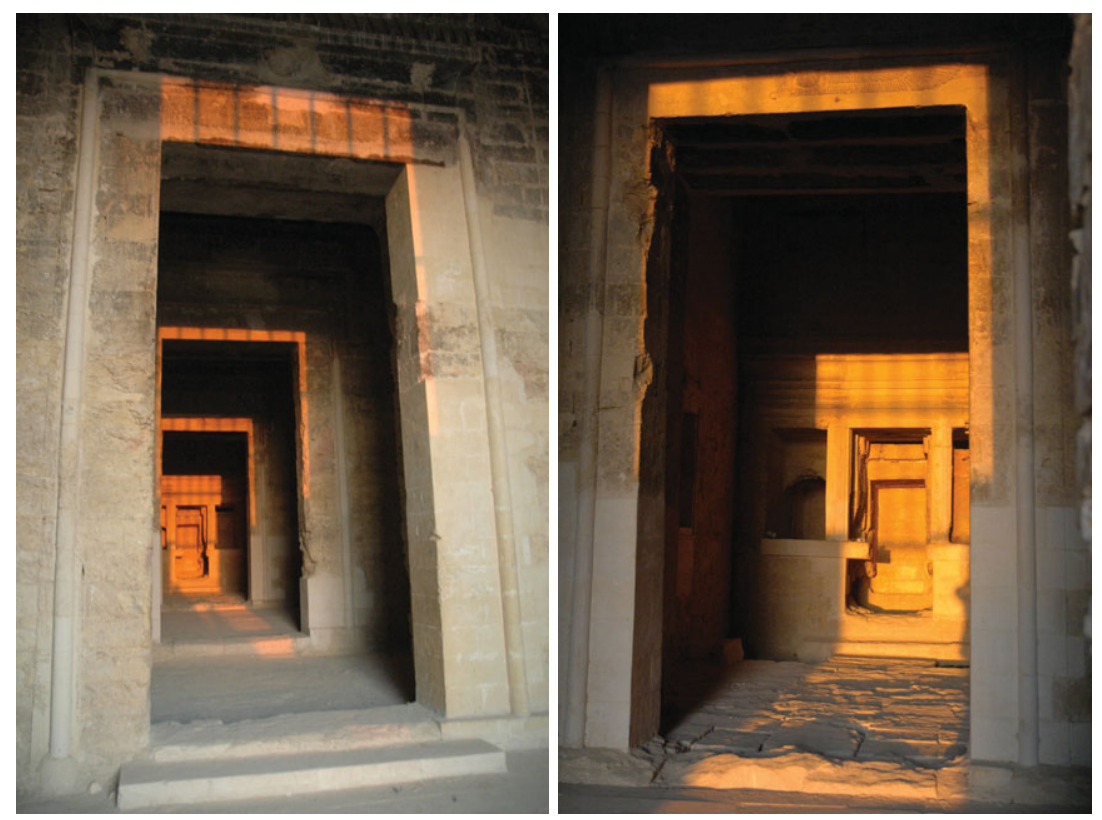

Figure 4. A splendid light hierophany at sunrise at the winter solstice (December 21st 2006) at the temple of Sobek-Re in Qsar Qarun (Dionysas) in El-Fayum Oasis. Notice the illumination of the inner sanctuary (left), where the statue of the god would have been located, and of the various successive portals. As the Sun rises, its light moves to the north illuminating the small shrine to the right of the sanctuary while the niche to its left, where the mummy (the underworld aspect) of the god was presumably located, always remains in darkness. Notice the parallelism with the illumination phenomenon reported for Abu Simbel. Photograph (c) J. A. Belmonte.

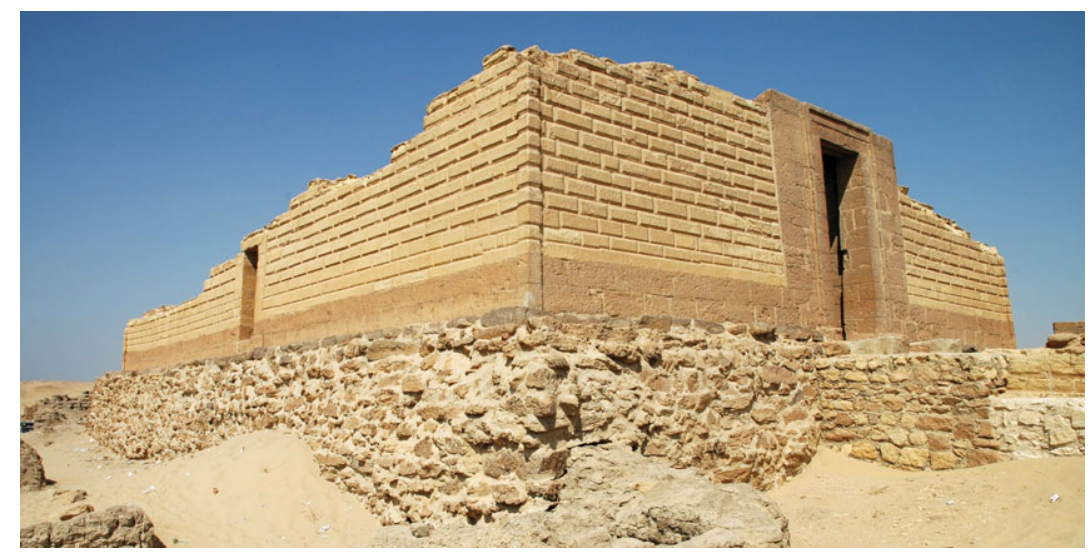

Figure 5. The south temple of Karanis, a distinguished member of Family III. An inscription of the temple is dated to the reign of the Roman Emperor Nero. However, according to our hypothesis, the temple may have been firstly orientated to sunrise at Wepet Renpet in c. 15 B.C. under Augustus. Photograph (C) J. A. Belmonte.

for 9 and 1, Panels (b), respectively. These values do not fit either the astronomical equinox or the mid-day between the solstices, the so-called megalithic equinox (see e.g. Ruggles 1997; González García \& Belmonte 2007).

However, considering this possibility, we suggest that primarily a Meridian axis would have been established for the pyramid complex and then the gate of the temple would have 


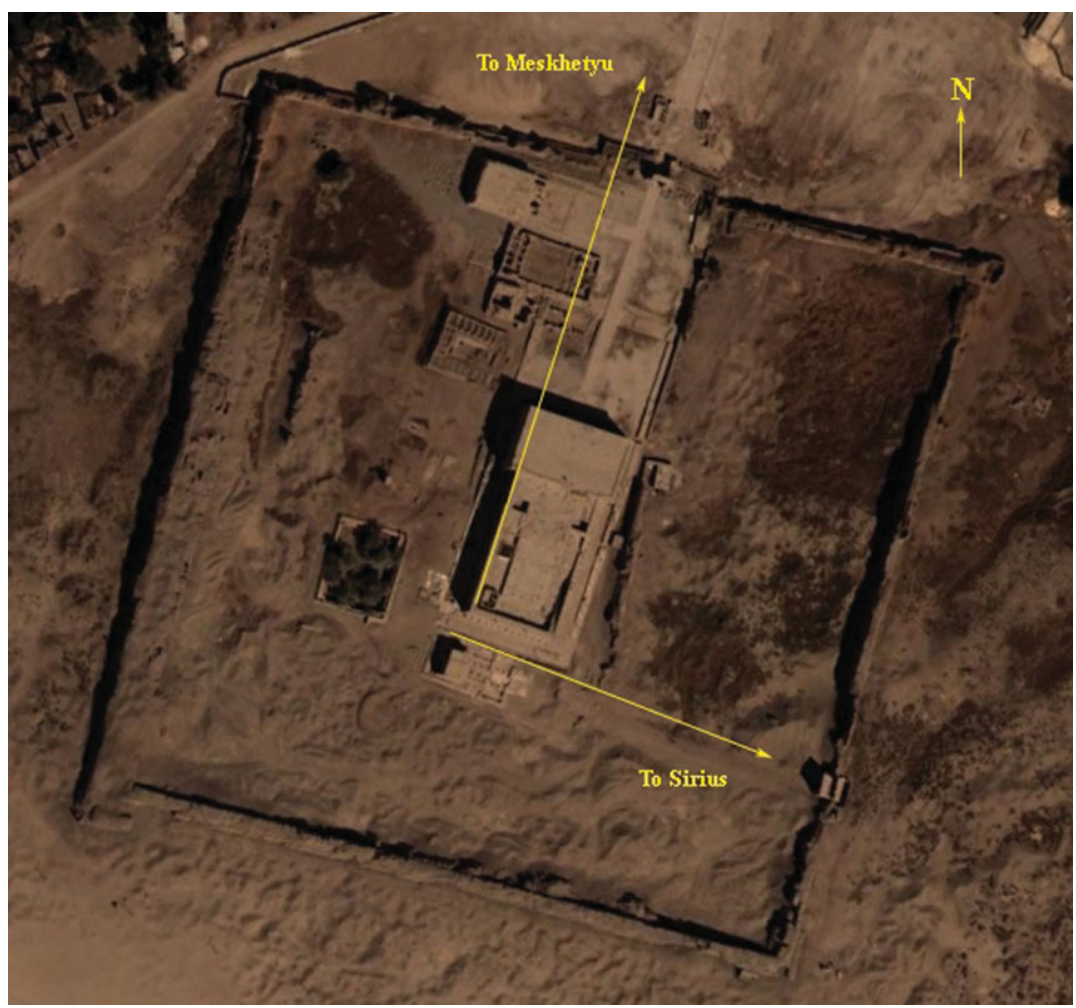

Figure 6. The complex of the temple of Hathor at Dendara showing a double alignment to Families VI and IV. The main building is orientated close to north and possibly to the rising of Meshketyu in the late Ptolemaic period. However, the hypostyle hall of the small Isis temple located behind it is not exactly perpendicular and could have been orientated to the rising of Sothis (Sopdet) as many other buildings on site before it. See Belmonte, Shaltout \& Fekri (2009) for further details. Adapted from an image courtesy of Google Earth.

been open to the east afterwards, in order to face sunrise and possibly to accommodate the topographical requirements of the Nile. This idea would be reinforced by the fact that, in later periods and, especially, for other geographical areas, eastern orientations were either imprecise or simply absent. The solar temples of the 5th Dynasty would be a particular exception since the original intention of their orientation may have been to face sunrise at Wepet Renpet (Egyptian New Year's Eve) at the moment of their construction (Belmonte, Shaltout \& Fekri 2009). Besides, seasonal orientations (family III, predominantly associated to peak III-) were present in all periods and geographical areas.

Family II is associated with the winter solstice and it is ubiquitous both in time and space. This demonstrates its importance. It is dominant during the New Kingdom and in Upper Egypt. Curiously, there is a peak related to summer solstice (II+) both in Fig. 9 (Panel $b$ ) and Fig. 2 (Panel $d$, Oases). We propose that the former may be associated with an interest in this time-marker during the Old Kingdom that might be related, among other aspects, to the foundation of the civil calendar. The latter would express the general interest in solar orientations in the outskirts of the Nile Valley. However, there is one peculiar and striking fact of the peaks related to Family II in Fig. 9. We have obtained values of $-24.5 \pm 0.75,-24.25 \pm 0.75$ and $-23.5 \pm 0.75$ for the peaks 


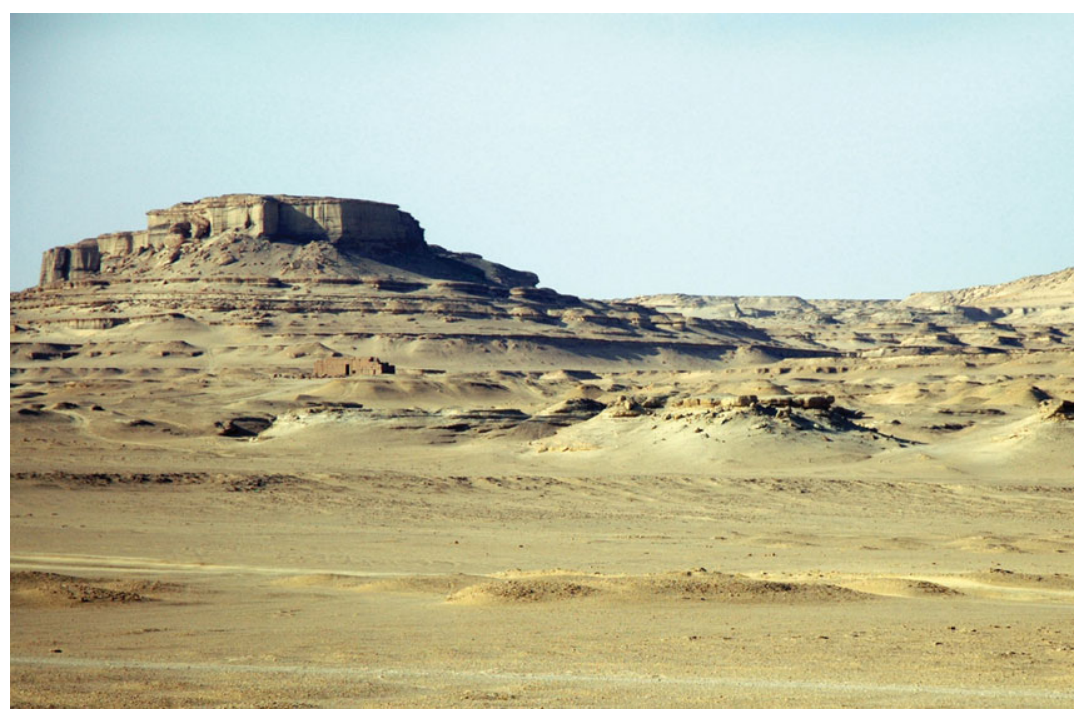

Figure 7. General view of the splendid landscape surrounding the megalithic temple of Qsar al-Sagha, located in the former northern shore of Lake Moeris and now ten kilometres inland inside the desert. This building could have been orientated to Canopus (Family V) at the moment of its construction c. 1840 BCE. Photograph (c) J. A. Belmonte.

of the earliest $(b)$, middle $(c)$ and latest $(d)$ periods, respectively. All these values are in agreement, within the errors, with the extreme values of the solar declination in the respective periods. However, we have detected a certain trend towards lower declinations (in absolute values) versus time which perhaps, and this is quite speculative, may be related to the decreasing value of the Ecliptic obliquity during Egyptian history. The obliquity of the Ecliptic is diminishing at a rate of $\sim 0.46$ seconds of arc per year. It varied from some $24^{\circ}$ to $23^{\circ} 37^{\prime}$ from 2900 to 1 BCE. If we are to believe the figures yielded by our solar alignments (of families I and II, fundamentally), one might suggest that our solar orientations were obtained when the disc was completely above the horizon. Indeed, this would not mean that ancient Egyptian were familiar with this physical phenomenon. The trend would simply be a collateral effect of their continuous interest in the phenomenon across time and the orientation of temples accordingly.

Family IV has two peculiarities. On the one hand, it is not apparently present in the oldest monuments, on the other, it is absent from the temples of the deserts and oases. We suggest that the explanation for the former could rely on the lesser importance, in certain aspects, of Sirius (Sopdet) in the earliest phases of Egyptian history. In the second case, the solution may result from the fact that Sopdet was most important because of her connection with the arrival of the waters of the Inundation. In lands where this phenomenon was not present, and thus irrelevant, temples orientated to this prominent star were not so mandatory. The family of Canopus (V) has a similar behaviour, being absent also in the earliest phases. Perhaps the absence of individual stellar alignments for these period could be related to the overwhelming dominance of cardinal (I, VI and VII) and solar (II, III and again I) orientations in those periods, reflecting different aspects of the stellar eschatology and the solarisation process of the kings in these earlier phases of Egyptian history. The Pyramid Texts are crystal-clear in this respect. The Sun (or the solar bark) and the Imperishable Stars (the Ikhemu Seku of ancient Egyptians of which 


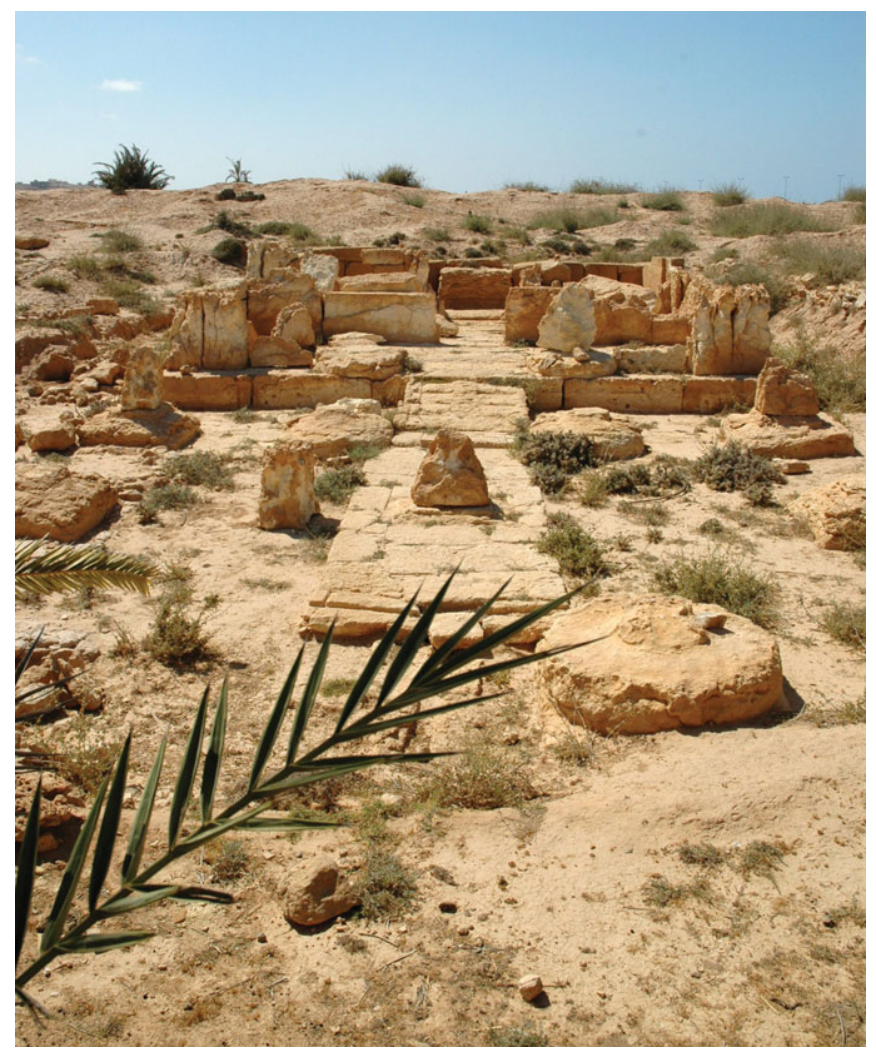

Figure 8. The temple of Ramses II at the border fortress of Zawiyet Umm el-Rakhmam (c. 1270 B.C.). Orientated to an azimuth of $133^{\circ}$, almost parallel to the contemporary Ramesseum $700 \mathrm{~km}$ distant, this would be a good example of the inter-cardinal family (VII). Photograph (c) J. A. Belmonte.

Meskhetyu was a fine example) are frequently mentioned as celestial destinies for the soul of the king after death.

The cardinal family (VI) is present during all periods of Egyptian history and in most areas of the country. Northern orientations (VI+) are dominant to southern counterparts, a fact presumably reflecting that $\mathrm{N}-\mathrm{S}$ orientations were predominantly, if not exclusively, traced to the north. Curiously, we have only an insignificant presence of the family in Lower Egypt, where eastern (cardinal) orientations are predominant. However, this could be related to the fact already mentioned that most eastern cardinal orientations would have originated as northern cardinal orientations.

Finally, our analysis shows two peculiarities for the quarter-cardinal family (VII). On the one hand, it is most typical of the New Kingdom and the early Late Period, when it is only second to the solstitial family and, in a certain aspect, substitutes family VI which is scarcely represented. On the other hand, it is practically exclusive to Upper Egypt. This could relate to the fact that this procedure of orientation was developed simultaneously to accomplish stellar and river orientation requirements, in the Nile Valley, as demonstrated in Paper 4.

As a summary of our exercise on evolution in time-and-space, we might reach the conclusion that actually only three conventions of orientation were present in ancient Egypt throughout her land and her history: cardinal (i), solar (ii) and stellar (iii). 


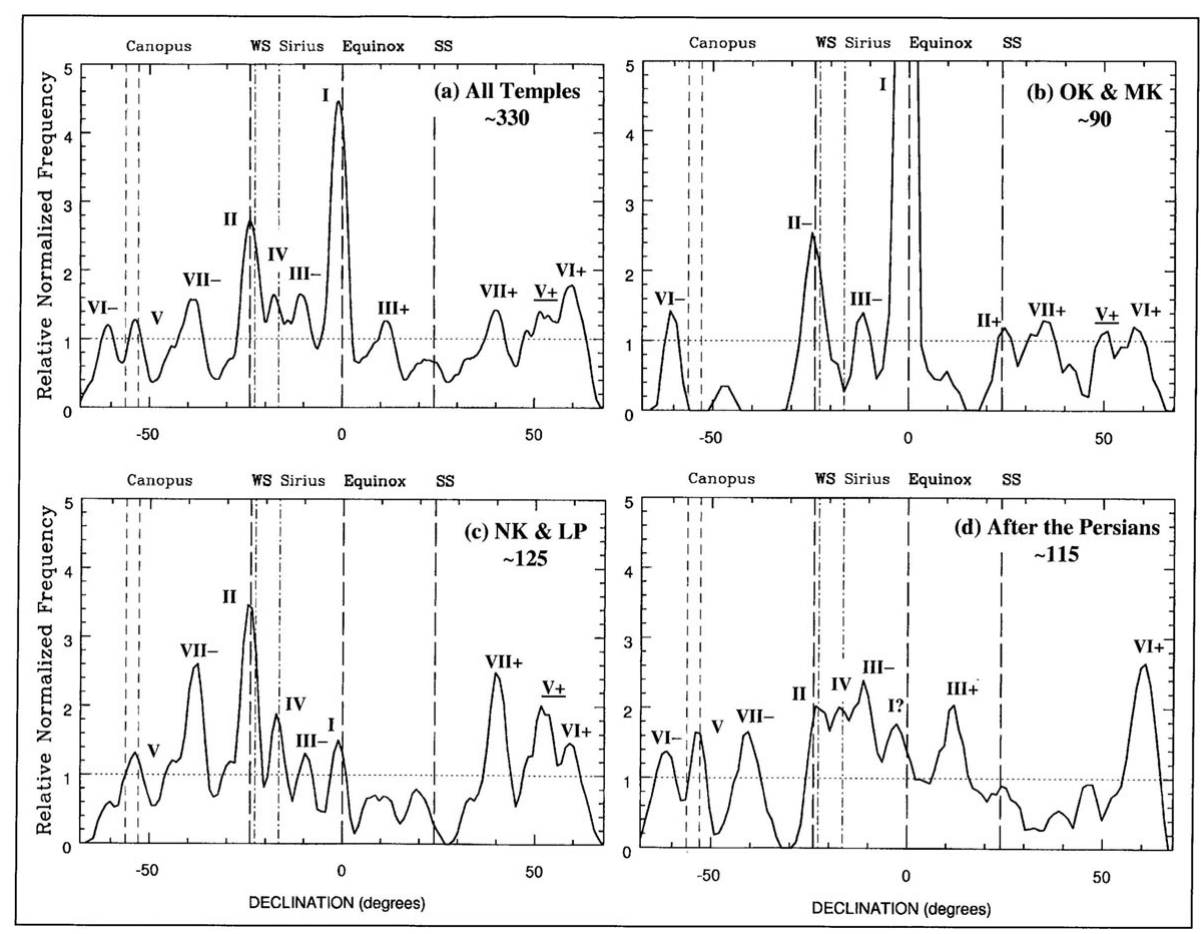

Figure 9. Histogram of declinations of the temples of ancient Egypt versus historical period. (a) Total histogram presented for comparison. (b) Temples from the pre-Dynastic period to the end of the Middle Kingdom. (c) Temples of the New Kingdom and the Late Period until the Persian conquest. (c) Late temples with a dominance of buildings of the Graeco-Roman period. The three series of data plotted in panels $(b),(c)$ and $(d)$ are independent of each other and are statistically significant. Notice the persistence of several families of orientation across time and the fluctuations of some others. In panel $(b)$, the peak of family I rises to more than 12 but has been cut to keep the same scale in the different plots.

- The cardinal convention would be incorporated by families I (in most occasions), VI and VII and would be achieved by the observations of certain configurations of stars in the north (predominantly, if not exclusively, stars of Meskhetyu). This procedure would initially give a near Meridian axis that would later offer various alternatives: a gate opening north, a gate opening south, a gate opening east (or west) or a new axis by turning the original by $45^{\circ}$ or $135^{\circ}$, with the gate opening near NE (or NW) or SE (or SW), respectively.

- The solar convention is formed by families I (in a few occasions), II, II $\perp$ and III and would basically be related to important points of the annual cycle, or in some cases to special dates in the civil calendar such as Wepet Renpet or the eves of the other two seasons of this calendar, Peret and Shemu.

- The stellar convention would be represented by families IV and V. We have no doubts of the pertinence and the relevance of the alignments to Sopdet. However, we have minor doubts as to whether many of the presumed alignments to Canopus ought to be interpreted in a different way. In this case, it is problematic whether new field data can provide a final answer. Hence, new epigraphic information confirming the importance of this star is highly desirable.

A final point to discuss is how once an alignment was provided by astronomical observations in a certain direction, the new axes at $45^{\circ}, 90^{\circ}, 135^{\circ}$ (in both clock-wise or 
anti-clockwise directions) or $180^{\circ}$ were obtained. The answer to this question may be found in the idea that the sign of Seshat (the divinity mostly involved in temple orientation ceremonies, notably the stretching of the cord), carried by the goddess upon her head in all representations, might perhaps have been a schematic and symbolic representation of an archaic transit instrument, similar to a Roman groma, that would have later become the emblem of the goddess (Belmonte, Molinero \& Miranda 2009). This instrument would have had eight radii and a viewpoint, and could have been used at the stretching of the cord ceremonies since the dawn of Egyptian history, directly offering the eight directions under discussion from a single astronomical or topographical observation.

\section{Conclusions: astronomy and culture}

Following the five field campaign carried out in the land of Egypt so far, we have accomplished some of the most relevant objectives that our research team had in mind for our archaeoastronomy project of ancient Egyptian culture. The principal dilemma we wanted to solve was whether the temples of this civilization were astronomically orientated or not. Epigraphic sources clearly mentioned solar and stellar targets as the references for temple orientations. However, the scientific community only agreed on the planning of orientations according to the Nile and the relevant inscriptions were sometimes considered, as in the case of Edwards (1993), as mere remembrance of long forgotten practices. We have now measured more than 330 temples and shrines throughout the geography of Egypt belonging to all periods of her history. This is an undoubtedly representative sample of all the temples in any state of preservation still existing in the country and it is indeed statistically significant.

I shall not go into details here about the many fascinating discoveries we have made in the course of this research (for a comprehensive summary, please check Belmonte, Shaltout \& Fekri 2009). However, we want to stress a few particular results that are real highlights of our work as seen from the astronomical point of view. These are:

- Ancient Egyptian temples were astronomically orientated beyond any reasonable doubt as all the analyses we have done with our data fully demonstrate.

- Among astronomical orientations, there were three, and only three, kind of targets. One was probably related to different celestial configurations of the stars of Meskhetyu in order to get a near or accurate Meridian orientation. This primary axis may have been rotated later by an eighth, a quarter or half a circumference to obtain any possible cardinal or quarter-cardinal direction (families I, VI and VII). The second had a markedly solar character and was fundamentally related to important time-marks of the annual cycle and/or the civil calendar (families I, II, II $\perp$ and III). Finally, the third group of targets includes the two brightest stars of ancient Egyptian skies, Sirius and Canopus (families IV and V, respectively). These customs were present during most of Egyptian history and in different areas of the country, although some minor peculiarities have been discovered. For example, solar orientations were transformed at certain historical periods with a view to orientating buildings to the beginning of the seasons of the civil calendar, notably Wepet Renpet, the First Day of the first month of the season of the Inundation, I Akhet 1 or New Year's Eve (see Fig. 5).

- Three families (I, VI and VII) would integrate a super-family of the so-called cardinal orientations. This would have basically consisted of the determination of a near Meridian (North-South) axis, for the general layout of a certain sacred structure, through the observation of some of the most conspicuous Imperishable Stars near the celestial pole, notably Meskhetyu. Once the Meridian alignment was determined, the monument could 
have opened east or west (family 1), north or south (family VI), or had its axis rotated through another $45^{\circ}$ (family VII). The pyramid complexes of the Old and Middle Kingdom are the paradigmatic examples of families I (the temples) and VI (the pyramids themselves).

- We have detected certain evidences of the precession of the equinoxes (see Paper 4) and of the variation of the obliquity of the Ecliptic phenomena in our data as collateral effects of the continuous use of stellar and solar orientations, respectively, during the course of Egyptian history. However, this marginal detection should never be interpreted under any circumstance as representing a real recognition of any of these phenomena by the ancient Egyptians.

Indeed, more work could be done and we are in the process of yielding new suggestive results as obtained from a Sixth field campaign in Middle Egypt and the analysis of Sudanese data (Belmonte et al. 2010). However, the author considers the sample presented in this work to be statistically representative beyond any doubt and he is convinced that new data will only serve to reinforce or faintly modify the conclusions. In fact, the work summarized here clearly illustrates something that we could only have imagined at the very outset of our project: the ancient Egyptians undoubtedly scrutinized the sky seeking their correct orientation not only in time, but also in space in their permanent search for cosmic order.

\section{Acknowledgements}

This work was financed in the framework of the projects P310793 Arqueoastronomía of the Instituto de Astroføisica de Canarias, AYA2004-01010 Orientatio ad Sidera of the Spanish MEC, and AYA2007-60213 Orientatio ad Sidera II of the Spanish MCINN.

\section{References}

Badawy, A. 1968, A History of Egyptian Architecture. III The Empire or New Kingdom (Berkeley: University of California Press)

Belmonte, J. A. 2009, in In search of cosmic order, selected essays on Egyptian archaeoastronomy, J.A. Belmonte and M. Shaltout (eds) (Cairo: American University in Cairo Press), 73

Belmonte, J. A. \& Shaltout, M. 2006, Journal for the History of Astronomy, 37, 173 (Paper 2)

Belmonte, J. A., Shaltout, M., \& Fekri, M. 2008, JHA, 39, 181 (Paper 4)

Belmonte, J. A., Molinero, M. A., \& Miranda, N. 2009, in In search of cosmic order, selected essays on Egyptian archaeoastronomy, J. A. Belmonte and M. Shaltout (eds) (Cairo: American University in Cairo Press), 193

Belmonte, J. A., Shaltout, M., \& Fekri, M. 2009, J. A. Belmonte, M. Shaltout and M. Fekri, in In search of cosmic order, selected essays on Egyptian archaeoastronomy, J. A. Belmonte and M. Shaltout (eds) (Cairo: American University in Cairo Press), 211

Belmonte, J. A., Fekri, M., Abdel-Hadi, Y. A., Shaltout, M., \& González Garcua, A. C. 2010, Journal for the History of Astronomy, 41, 65

Edwards, I. E. S. 1993, The Pyramids of Egypt (Harmondsworth: Penguin)

González García, A. C. \& Belmonte, J. A. 2007, Archaeoastronomy, the Journal for Astronomy in Culture, 20, 97

Krauss, R. 1997, Astronomische Konzepte und Jenseitsvorstellungen in den Pyramidentexten, Ägyptologische Abhandlung Band 59 (Wiesbaben: Harrasowitz)

Lull, J. \& Belmonte, J. A. 2006, Journal for the History of Astronomy, 37, 373

Lull, J. \& Belmonte, J. A. 2009, in In search of cosmic order, selected essays on Egyptian archaeoastronomy, J. A. Belmonte and M. Shaltout (eds) (Cairo: American University in Cairo Press), 153 
Maravelia, A. A. 2006, Les Astres dans les Textes Religieux en Egypte Antique et dans les Hymnes Orphiques BAR International Series 1527 (Oxford: Archaeopress)

Ruggles, C. L. N. 1997, Archaeoastronomy, 22, S45

Shaltout, M. \& and Belmonte, J. A. 2005, JHA, 36, 273 (Paper 1)

Shaltout, M., Belmonte, J. A., \& Fekri, M. 2007, JHA, 38, 141 (Part I) and 413 (Part II) (Paper 3)

Spence, K. 2000, Nature, 408, 320

Wilkinson, R. H. 2000, The complete temples of ancient Egypt (London: Thames \& Hudson) 